\title{
SYNTHESIS OF 2-(2-(HYDROXYMETHYL)PHENYL)ETHANOL DERIVATIVES AS POTENTIAL ANTIBACTERIAL AGENTS
}

\author{
P. MANIVEL ${ }^{a, b}$, F. NAWAZ KHAN ${ }^{a^{*}}$ \\ ${ }^{a}$ Organic Chemistry Division, School of Science and Humanities, VIT University, Vellore, Tamil Nadu, India \\ ${ }^{b}$ Syngene International Limited, Bangalore, Karnataka, India. \\ (Received: July 28, 2008 - Accepted: April 11, 2009)
}

\begin{abstract}
Reaction of 3-substituted isocoumarins (1a-h) with excess of sodium borohydride in methanol gave the corresponding 2-(2-(hydroxymethyl)phenyl)ethanol derivatives (2a-h). Antimicrobial activities of synthesized compounds were measured, using Gram-negative (Escherichia coli, Salmonella typhi, Proteus mirabilis) and Gram-positive bacteria (Bacillus cereus, Staphylococcus aureus).
\end{abstract}

Key words: Isocoumarin, sodium borohydride, diol, antimicrobial properties.

\section{INTRODUCTION}

Synthesis of variety of compounds like carbocyclic, heterocyclic compounds and various aromatic compounds can be effected from isocoumarins intermediates. ${ }^{1}$ The hydroxyl structural moiety was found in numerous pharmaceutically active compounds and therefore represents an interesting template for combinatorial as well as medicinal chemistry. ${ }^{2}$ In particular phenylethanol derivatives have good antifungal properties.3, 4 An increasing number of new isocoumarins in nature and increasing importance of diol derivatives have stimulated our researcher group a continued interest for synthesis of 2-(2-(hydroxymethyl)phenyl)ethanols from the precursor isocoumarins. Recently, several methods have been reported for the synthesis of diols such as palladium catalyzed reactions, electrophilic aromatic substitution, cyclization of 2-allyl- and alkenyl benzoic acid, etc. ${ }^{5-9}$ In continuous of research interests, ${ }^{10-20}$ present investigation aimed at simplified reaction of isocoumarins and sodium borohydride to the corresponding 2-(2-(hydroxymethyl)phenyl) ethanol derivatives without isolation of intermediate dihydroisocoumarins. (Scheme 1)
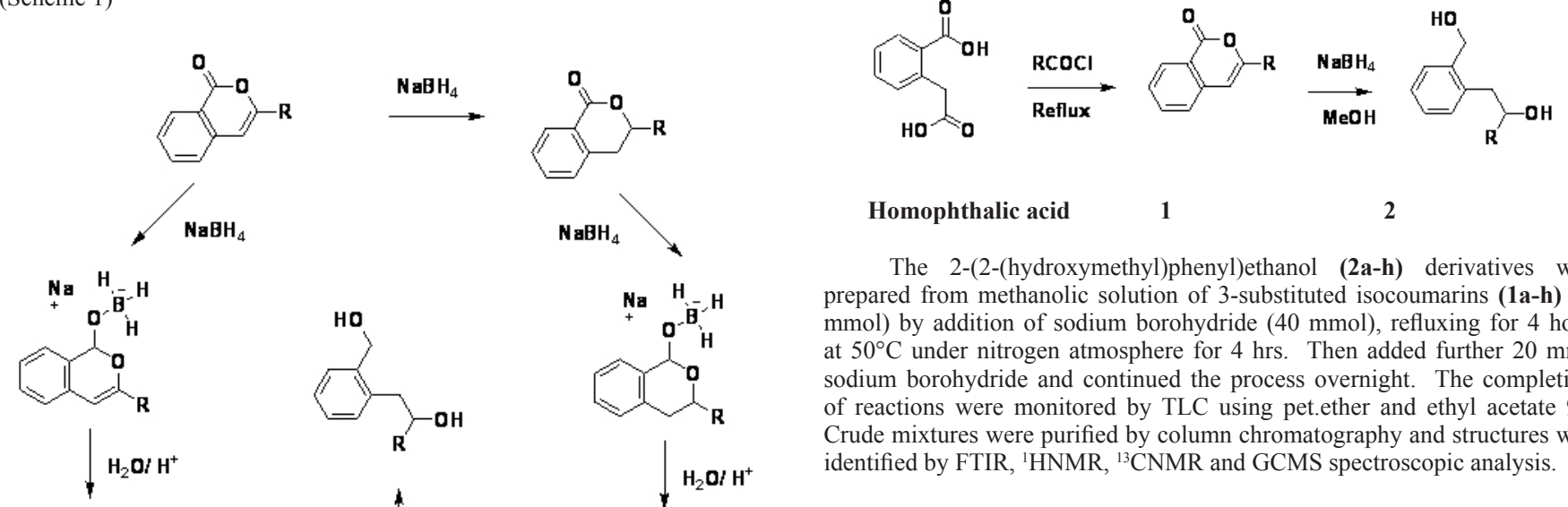

Homophthalic acid

1

The 2-(2-(hydroxymethyl)phenyl)ethanol (2a-h) derivatives were prepared from methanolic solution of 3-substituted isocoumarins (1a-h) $(10$ $\mathrm{mmol}$ ) by addition of sodium borohydride $(40 \mathrm{mmol})$, refluxing for 4 hours at $50^{\circ} \mathrm{C}$ under nitrogen atmosphere for $4 \mathrm{hrs}$. Then added further $20 \mathrm{mmol}$ sodium borohydride and continued the process overnight. The completions of reactions were monitored by TLC using pet.ether and ethyl acetate 9:1. Crude mixtures were purified by column chromatography and structures were identified by FTIR, ${ }^{1} \mathrm{HNMR},{ }^{13} \mathrm{CNMR}$ and GCMS spectroscopic analysis.

Synthesis of 1-(2-(hydroxymethyl)phenyl)hexan-2-ol (2a) from 3-nButyl isocoumarin (1a)

3-n-Butylisocoumarin, 1a (1 eq.) was dissolved in 10 volumes of methanol, sodium borohydride (4 eq.) was added to it and stirred at $50^{\circ} \mathrm{C}$ under nitrogen atmosphere for $4 \mathrm{hrs}$, then two more equivalents of $\mathrm{NaBH}_{4}$ was further added and left overnight at $50^{\circ} \mathrm{C}$ for completion of reaction. After TLC analysis, solvent methanol was removed, residue added to water and extracted with ethyl acetate. Ethyl acetate layer was washed with water, dried with anhydrous $\mathrm{Na}_{2} \mathrm{SO}_{4}$, evaporated to yield the product diol, 2a, which was further purified by washing with petroleum ether. The product was characterized by NMR, GCMS techniques.

Similar procedures were followed for the synthesis of other phenylethanol derivatives $\mathbf{2 b}-\mathbf{h}$ and the results have been tabulated as Table 1 .

\section{RESULTS AND DISCUSSION}

In this work we report synthesis of potential antibacterial diol derivatives 
containing the phenylethanol structural moiety. Thus, the reaction between the isocoumarins (1) and sodiumborohydride in methanol at $50^{\circ} \mathrm{C}$ gave a single product (2). The structure of $\mathbf{2}$ was confirmed on the basis of IR spectrum which showed the absence of any $\mathrm{C}=\mathrm{O}$ and $\mathrm{C}=\mathrm{C}$ stretching of starting material isocoumarin, IR spectra of diols showed peak values at 3400-3070 (due to $\mathrm{OH}$ ), 3000- 3080 (due to Arm CH) 1500 - 1420 (due to $\mathrm{C}=\mathrm{CH}$ ), 1019 (due to $\mathrm{C}-\mathrm{O}$ ). GCMS analysis of diols formed in the reduction of isocoumarins have shown mass peaks at $\mathrm{m} / \mathrm{e} \mathrm{M}^{+}-18$ peaks, base peak at $\mathrm{m} / \mathrm{e} 104$ for all compounds, $\mathbf{2 a - 2 h}$ corresponding to the water elimination and $\mathrm{C} 6 \mathrm{H} 4-\mathrm{CO}$ respectively along with other fragmentation peaks. The present paper also included NMR characterization of these compounds, $\mathbf{2 a - 2 h}$.

\section{Analysis Data}

1-(2-(hydroxymethyl)phenyl)hexan-2-ol, 2a, Gummy solid, IR (KBr) v $3323(\mathrm{OH}), 3064,3020,2850,1455,1424(\mathrm{C}=\mathrm{C}), 1011 \mathrm{~cm}^{-1}(\mathrm{C}-\mathrm{O})$; ' ${ }^{1} \mathrm{H}$ NMR $\left(400 \mathrm{MHz}\right.$, DMSO $\left.-\mathrm{d}_{\mathrm{f}}\right): \delta 7.33(\mathrm{q}, \mathrm{J}=2.98 \mathrm{~Hz}, 1 \mathrm{H}), 7.15(\mathrm{~d}, \mathrm{~J}=2.60 \mathrm{~Hz}$, $3 \mathrm{H}), 5.07(\mathrm{t}, \mathrm{J}=5.42 \mathrm{~Hz}, 1 \mathrm{H}), 4.59-4.46(\mathrm{~m}, 3 \mathrm{H}), 3.59-3.56(\mathrm{~m}, 1 \mathrm{H}), 2.64$ $(\mathrm{t}, \mathrm{J}=3.64 \mathrm{~Hz}, 2 \mathrm{H}), 1.36(\mathrm{~m}, \mathrm{~J}=4.63 \mathrm{~Hz}, 2 \mathrm{H}) 1.23(\mathrm{~m}, \mathrm{~J}=6.74 \mathrm{~Hz}, 4 \mathrm{H})$, $0.84(\mathrm{t}, \mathrm{J}=7.06 \mathrm{~Hz}, 3 \mathrm{H}) ;{ }^{13} \mathrm{C}$ NMR $\left(100 \mathrm{MHz}, \mathrm{DMSO}-\mathrm{d}_{6}\right) \delta 140.70,137.92$, 130.55, 127.89, 127.01126 .09 (Aromatic carbons), 71.29, 61.37, 2 X37.37, 28.01, 22.72 (Aliphatic carbons), 14.50; GCMS- 190 (M-18); $\mathrm{C}_{13} \mathrm{H}_{20} \mathrm{O}_{2}$ Mol. Wt.: 208.3, Calculated C, 74.96; H, 9.68; O, 15.36 Found C, 74.92; H, 9.17; $\mathrm{O}, 15.34 \%$

2-(2-(hydroxymethyl)phenyl)-1-phenylethanol, $2 \mathrm{~b}$ Colourless solid, $\mathrm{mp}$ $90^{\circ} \mathrm{C}, \mathrm{IR}(\mathrm{KBr}) \vee 3238(\mathrm{OH}), 3024,2850,1474,1424(\mathrm{C}=\mathrm{C}), 1325,1201$, 1057 (C-O), 950, 758, $702 \mathrm{~cm}^{-1} .{ }^{1} \mathrm{H}$ NMR (400 MHz, DMSO - d $): \delta 7.32-7.22$ $(\mathrm{m}, 5 \mathrm{H}), 7.15(\mathrm{~d}, \mathrm{~J}=5.64 \mathrm{~Hz}, 4 \mathrm{H}), 5.37(\mathrm{~d}, \mathrm{~J}=4.52 \mathrm{~Hz}, 1 \mathrm{H}), 5.09(\mathrm{t}, \mathrm{J}=5.34$ $\mathrm{Hz}, 1 \mathrm{H}), 4.74(\mathrm{~m}, \mathrm{~J}=4.42 \mathrm{~Hz}, 1 \mathrm{H}), 4.44(\mathrm{~m}, \mathrm{~J}=7.43 \mathrm{~Hz}, 2 \mathrm{H}), 2.89(\mathrm{t}, \mathrm{J}=6.76$ $\mathrm{Hz}, 2 \mathrm{H}) .{ }^{13} \mathrm{C}$ NMR $\left(100 \mathrm{MHz}, \mathrm{DMSO}-\mathrm{d}_{\mathrm{f}}\right) \delta 146.55,140.84,137.37,130.80$, $128.37,2 \mathrm{X} 128.01,2 \mathrm{X} 127.22,127.01,126.31,126.28$ (Aromatic carbons), $73.94,61.55,42.60$ (Aliphatic carbons). GCMS- $210(\mathrm{M}-18), \mathrm{C}_{15} \mathrm{H}_{16} \mathrm{O}_{2}$ Mol. Wt.: 228.29, Calcuated C, 78.92; H, 7.06; O, 14.02, Found C, 78.65; H, 6.92; $\mathrm{O}, 13.98 \%(\mathrm{OH}),(\mathrm{C}=\mathrm{C}),(\mathrm{C}-\mathrm{O})$

2-(2-(hydroxymethyl)phenyl)-1-p-tolylethanol, 2c Colourless solid, mp $76^{\circ} \mathrm{C}, \mathrm{IR}(\mathrm{KBr}) \vee 3187(\mathrm{OH}), \quad, 3016,2917,1934,1475,1451(\mathrm{C}=\mathrm{C}), 1308$, 1204, 1062 (C-O), 814, 767, $712 \mathrm{~cm}^{-1} ;{ }^{1} \mathrm{H}$ NMR (400 MHz, DMSO - d $): \delta$ $7.31(\mathrm{~d}, \mathrm{~J}=5.36 \mathrm{~Hz}, 1 \mathrm{H}), 7.20-7.08(\mathrm{~m}, 7 \mathrm{H}), 5.29(\mathrm{~d}, \mathrm{~J}=4.32 \mathrm{~Hz}, 1 \mathrm{H}), 5.08(\mathrm{t}$, $\mathrm{J}=5.18 \mathrm{~Hz}, 1 \mathrm{H}), 4.69(\mathrm{~d}, \mathrm{~J}=6.04 \mathrm{~Hz}, 1 \mathrm{H}), 4.51(\mathrm{q}, \mathrm{J}=5.90 \mathrm{~Hz}, 1 \mathrm{H}), 4.42(\mathrm{q}, \mathrm{J}=$ $6.18 \mathrm{~Hz}, 1 \mathrm{H}), 2.85(\mathrm{q}, \mathrm{J}=8.16 \mathrm{~Hz}, 2 \mathrm{H}), 2.27(\mathrm{~s}, 3 \mathrm{H}) \cdot{ }^{13} \mathrm{C}(100 \mathrm{MHz}, \mathrm{DMSO}-$ $\left.\mathrm{d}_{6}\right) \delta 143.54,140.79,2 \mathrm{X} 137.38,136.08,130.73,2 \mathrm{X} 128.88,127.92,2 \mathrm{X} 126.95$, 126.23 (Aromatic carbons), 73.71, 61.45, 42.57, 21.17 (Aliphatic carbons). GCMS- 224 (M-18), $\mathrm{C}_{16} \mathrm{H}_{18} \mathrm{O}_{2}$, Mol. Wt.: 242.31, Calculated C, 79.31; H, 7.49; O, 13.21, Found C, 78.70; H, 7.19; O, 13.11 .

1-(4-chlorophenyl)-2-(2-(hydroxymethyl)phenyl)ethanol, 2d Colorless solid, $\mathrm{mp} 104^{\circ} \mathrm{C}$, IR $(\mathrm{KBr}) \vee 3244(\mathrm{OH}), 3018,2853,1490,1422(\mathrm{C}=\mathrm{C}), 1325$, $1212,1061(\mathrm{C}-\mathrm{O}), 1000,772,742 \mathrm{~cm}^{-1}$; ${ }^{1} \mathrm{H}$ NMR $\left(400 \mathrm{MHz}, \mathrm{DMSO}-\mathrm{d}_{6}\right): \delta$ 7.35- $7.11(\mathrm{~m}, 8 \mathrm{H}), 5-47-5.45(\mathrm{~d}, \mathrm{~J}=4.5 \mathrm{~Hz} 1 \mathrm{H}), 5.11-5.08(\mathrm{t}, \mathrm{J}=5.1 \mathrm{~Hz} 1 \mathrm{H})$, 4.78- $4.72(\mathrm{~m} \mathrm{1H}), 4.53-4.41(\mathrm{~m}, 2 \mathrm{H}), 2.94-2.82(\mathrm{~m}, 2 \mathrm{H}) \cdot{ }^{13} \mathrm{C} \mathrm{NMR}(100 \mathrm{MHz}$, DMSO - d $) \delta 145.47,140.83,136.99,131.59,130.82$, 2X128.28, 2X128.18, 128.04, 127.01, 126.38 (Aromatic carbons), 73.16, 61.51, 42.41 (Aliphatic carbons). GCMS- 244 (M-18), $\mathrm{C}_{15} \mathrm{H}_{15} \mathrm{ClO}_{2}$, Mol. Wt.: 262.73, Calculated C, 68.57; H, 5.75; O, 12.18, Found C, 68.23; H, 5.64; O, 12.12 .

2-(2-(hydroxymethyl)phenyl)-1-(4-methoxyphenyl)ethanol, $2 \mathrm{e}$ Colourless solid, mp $68^{\circ} \mathrm{C}$, IR $(\mathrm{KBr}) \vee 3245 .(\mathrm{OH}), 3006,2852,1511,1424$ $(\mathrm{C}=\mathrm{C}), 1325,1243,1061$ (C-O), 1005, 827, $760 \mathrm{~cm}^{-1} .{ }^{1} \mathrm{H}$ NMR $(400 \mathrm{MHz}$, DMSO - d $): \delta 7.30(\mathrm{~d}, \mathrm{~J}=5.12 \mathrm{~Hz}, 1 \mathrm{H}), 7.20(\mathrm{~d}, \mathrm{~J}=8.32 \mathrm{~Hz}, 2 \mathrm{H}), 7.12(\mathrm{~m}$, $3 \mathrm{H}), 6.84(\mathrm{~d}, \mathrm{~J}=8.08 \mathrm{~Hz}, 2 \mathrm{H}), 5.25(\mathrm{~d}, \mathrm{~J}=4.44 \mathrm{~Hz}, 1 \mathrm{H}), 5.07(\mathrm{t}, \mathrm{J}=5.32 \mathrm{~Hz}$, $1 \mathrm{H}), 4.68(\mathrm{~m}, \mathrm{~J}=4.36 \mathrm{~Hz}, 1 \mathrm{H}), 4.50(\mathrm{q}, \mathrm{J}=5.96 \mathrm{~Hz}, 1 \mathrm{H}), 4.41(\mathrm{q}, \mathrm{J}=6.22 \mathrm{~Hz}$, $1 \mathrm{H}), 3.71(\mathrm{~s}, 3 \mathrm{H}), 2.86(\mathrm{~m}, \mathrm{~J}=7.24 \mathrm{~Hz}, 2 \mathrm{H}) .{ }^{13} \mathrm{C}$ NMR $\left(100 \mathrm{MHz}, \mathrm{DMSO}-\mathrm{d}_{6}\right)$ $\delta 158.55,140.79,138.52,137.36,130.72,2 \times 127.89,127.38,126.93,126.21$, 2X113.70 (Aromatic carbons), 73.46, 61.44, 42.59 (Aliphatic carbons), 55.45 $\left(\mathrm{OCH}_{3}\right)$,. GCMS-240 (M-18), $\mathrm{C}_{16} \mathrm{H}_{18} \mathrm{O}_{3}$, Mol. Wt.: 258.31, Calculated C, 74.39; H, 7.02; O, 18.58, Found C,73.97; H, 7.03; O, 18.54 .

2-(2-(hydroxymethyl)phenyl)-1-(naphthalen-1-yl)ethanol,

2f Colourless solid, $\mathrm{mp} 142^{\circ} \mathrm{C}$, IR $(\mathrm{KBr}) \vee 3229 .(\mathrm{OH}), 3061,2852,1469,1448$ $(\mathrm{C}=\mathrm{C}), 1331,1229,1061(\mathrm{C}-\mathrm{O}), 994,791,747 \mathrm{~cm}^{-1}$. ${ }^{1} \mathrm{H}$ NMR $(400 \mathrm{MHz}$, DMSO $\left.-\mathrm{d}_{6}\right): \delta 8.27(\mathrm{~d}, \mathrm{~J}=8.16 \mathrm{~Hz}, 1 \mathrm{H}), 7.93(\mathrm{t}, \mathrm{J}=4.66 \mathrm{~Hz}, 1 \mathrm{H}), 7.81(\mathrm{~d}$, $\mathrm{J}=8.08 \mathrm{~Hz}, 1 \mathrm{H}), 7.63(\mathrm{t}, \mathrm{J}=3.54 \mathrm{~Hz}, 1 \mathrm{H}), 7.47(\mathrm{~m}, 3 \mathrm{H}), 7.34-7.33(\mathrm{~m} 1 \mathrm{H})$, 7.26- $7.15(\mathrm{~m}, 3 \mathrm{H}), 5.53(\mathrm{~d}, \mathrm{~J}=4.88 \mathrm{~Hz}, 2 \mathrm{H}), 5.12(\mathrm{t}, \mathrm{J}=5.36 \mathrm{~Hz}, 1 \mathrm{H}), 4.51$ $(\mathrm{m}, \mathrm{J}=5.94 \mathrm{~Hz}, 2 \mathrm{H}), 3.05(\mathrm{q}, \mathrm{J}=3.45 \mathrm{~Hz}, 2 \mathrm{H}) .{ }^{13} \mathrm{C} \mathrm{NMR}(100 \mathrm{MHz}, \mathrm{DMSO}-$ $\left.\mathrm{d}_{6}\right) \delta 142.23,140.91,137.58,133.73,130.67,130.47,129.07,128.12,127.64$, $127.08,126.32,126.29,125.85,125.82,123.89,123.78$ (Aromatic carbons), 70.90, 61.63, 41.43 (Aliphatic carbons). GCMS- 260 (M-18), $\mathrm{C}_{19} \mathrm{H}_{18} \mathrm{O}_{2}$, Mol. Wt.: 278.35, Calculated C, 81.99; H, 6.52; O, 11.50, Found C, 81.56; H, 6.45;
O, 11.46 .

1-(furan-2-yl)-2-(2-(hydroxymethyl)phenyl)ethanol, 2g Gummy solid, IR $(\mathrm{KBr}) \vee 3368(\mathrm{OH}), 3064,2852,1492,1451(\mathrm{C}=\mathrm{C}), 1010(\mathrm{C}-\mathrm{O}) \mathrm{cm}^{-1} .{ }^{1} \mathrm{H}$ NMR $\left(400 \mathrm{MHz}, \mathrm{DMSO}-\mathrm{d}_{\mathrm{s}}\right): \delta 7.56(\mathrm{~s}, 1 \mathrm{H}), 7.56(\mathrm{t}, \mathrm{J}=0.90 \mathrm{~Hz}, 1 \mathrm{H}), 7.33$ (t, J= 4.22 Hz, H), $7.12(\mathrm{~m}, 3 \mathrm{H}), 6.35(\mathrm{q}, \mathrm{J}=1.62 \mathrm{~Hz}, 1 \mathrm{H}), 6.20(\mathrm{~d}, \mathrm{~J}=3.12 \mathrm{~Hz}$, $1 \mathrm{H}), 5.43(\mathrm{~d}, \mathrm{~J}=5.48 \mathrm{~Hz}, 1 \mathrm{H}), 5.07(\mathrm{t}, \mathrm{J}=5.32 \mathrm{~Hz}, 1 \mathrm{H}), 4.71(\mathrm{~m}, \mathrm{~J}=3.88 \mathrm{~Hz}$, $1 \mathrm{H}), 4.51(\mathrm{~m}, \mathrm{~J}=5.75 \mathrm{~Hz}, 2 \mathrm{H}), 3.01(\mathrm{~m}, \mathrm{~J}=6.56 \mathrm{~Hz}, 2 \mathrm{H}) .{ }^{13} \mathrm{C} \mathrm{NMR}(100 \mathrm{MHz}$, DMSO - d $) \delta 158.11,142.02,140.85,136.64,130.54,127.95,127.00,126.39$, 110.60, 106.01 (Aromatic carbons), 67.44, 61.37, 38.73 (Aliphatic carbons). GCMS-200 (M-18), $\mathrm{C}_{13} \mathrm{H}_{18} \mathrm{O}_{3}$, Mol. Wt.: 218.25, Calculated C, 71.54; H, 6.47; $\mathrm{O}, 21.99$, Found $\mathrm{C}, 71.00 ; \mathrm{H}, 6.39 ; \mathrm{O}, 21.88$.

2-(2-(hydroxymethyl)phenyl)-1-(thiophen-2-yl)ethanol, 2h Gummy solid, IR (KBr) v $3342(\mathrm{OH})$, 3054, 2872, 1492, $1451(\mathrm{C}=\mathrm{C}), 1034(\mathrm{C}-\mathrm{O})$, 748, $699 \mathrm{~cm}^{-1} .{ }^{1} \mathrm{H}$ NMR $\left(400 \mathrm{MHz}, \mathrm{DMSO}-\mathrm{d}_{6}\right): \delta 7.37-7.32(\mathrm{~m}, 2 \mathrm{H}), 7.15$ $(\mathrm{d}, \mathrm{J}=6.32 \mathrm{~Hz}, 3 \mathrm{H}), 6.92(\mathrm{~m}, 1 \mathrm{H}),-6.84(\mathrm{~d}, \mathrm{~J}=3.12 \mathrm{~Hz}, 1 \mathrm{H}), 5.74(\mathrm{t}, \mathrm{J}=$ $3.28 \mathrm{~Hz}, 1 \mathrm{H}), 5.08(\mathrm{t}, \mathrm{J}=5.32 \mathrm{~Hz}, 1 \mathrm{H}), 4.98(\mathrm{~m}, \mathrm{~J}=4.57 \mathrm{~Hz}, 1 \mathrm{H}), 4.52(\mathrm{q}, \mathrm{J}=$ $6.02 \mathrm{~Hz}, 1 \mathrm{H}), 4.45(\mathrm{q}, \mathrm{J}=6.20 \mathrm{~Hz}, 1 \mathrm{H}), 2.98(\mathrm{~d}, \mathrm{~J}=6.68 \mathrm{~Hz}, 2 \mathrm{H}) .{ }^{13} \mathrm{C}$ NMR $\left(100 \mathrm{MHz}, \mathrm{DMSO}-\mathrm{d}_{6}\right) \delta 143.54,140.79,137.38,136.08,130.73,128.88$, 127.92, 2X126.95, 126.23 (Aromatic carbons), 73.71, 61.45, 42.57 (Aliphatic carbons). GCMS-216(M-18), $\mathrm{C}_{13} \mathrm{H}_{18} \mathrm{O}_{3}$, Mol. Wt.: 234.31, Calculated C, 66.64; H, 6.02; O, 13.66; S, 13.68, Found C, 66.52; H, 5.89; O, 13.54 .

\section{Antibacterial activity}

The in vitro antibacterial screening of synthesized compounds $\mathbf{2} \mathbf{a}-\mathbf{h}$ were evaluated against selected Gram-positive organisms viz. Bacillus cereus, Staphylococcus aureus and Gram-negative organisms viz. Escherichia coli, Salmonella typhi, Proteus mirabilis by Agar well diffusion method. ${ }^{21}$ Cultures of bacteria were grown on nutrient broth (HiMedia) at $37^{\circ} \mathrm{C}$ for $12-14 \mathrm{hr}$ and were maintained on respective agar slants at $4^{\circ} \mathrm{C}$. Nutrient agar was poured onto a plate and allowed to solidify. Test compounds (DMSO solutions) of $4 \mathrm{mg} / \mathrm{ml}$ concentration were used as stock solution from this 50 or $100 \mu \mathrm{l}$ was loaded to each well of $10 \mathrm{~mm}$ diameter. The plates were then kept at $5^{\circ} \mathrm{C}$ for 1 $\mathrm{h}$ then transferred to an incubator maintained at $36^{\circ} \mathrm{C}$. The width of the growth inhibition zone was measured after $24 \mathrm{~h}$ incubation. The activity studies have been carried out with two different concentration and triplicate measurements (Table 2).

Table 1.- Reduction of isocoumarins ${ }^{\mathrm{a}}$ using Sodium borohydride.
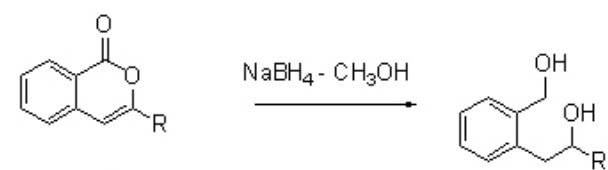

1

\begin{tabular}{|c|c|c|c|}
\hline & & and Product $\mathbf{2}$ & Yield \\
\hline Entry & & $\mathrm{R}$ & 70 \\
\hline 1 & $2 \mathbf{a}$ & & 84 \\
\hline 2 & $2 b$ & & 85 \\
\hline 3 & 2c & & 81 \\
\hline 4 & $2 d$ & & 74 \\
\hline 5 & $2 \mathrm{e}$ & & 77 \\
\hline 6 & $2 f$ & & 72 \\
\hline 7 & $2 \mathrm{~g}$ & & 79 \\
\hline 8 & $2 h$ & & 73 \\
\hline
\end{tabular}

aAll products were identified by ${ }^{1} \mathrm{H},{ }^{13} \mathrm{C} N M R, G C M S$ blsolated Yields 
Table 2.- Antimicrobial activity of synthesized compounds (Zones of inhibition in mm).

\begin{tabular}{|c|c|c|c|c|c|c|c|c|c|c|c|c|c|}
\hline \multirow{4}{*}{ Bacterial Strains } & \multicolumn{12}{|c|}{ Synthesized Compounds } & \multirow{3}{*}{$\begin{array}{r}\text { Streptomycin } \\
100 \mu \mathrm{I}\end{array}$} \\
\hline & \multicolumn{2}{|c|}{$2 \mathrm{a}(\mu \mathrm{L})$} & \multicolumn{2}{|c|}{$2 \mathrm{~b}(\mu \mathrm{L})$} & \multicolumn{2}{|c|}{$2 \mathrm{c}(\mu \mathrm{L})$} & \multicolumn{2}{|c|}{$2 \mathrm{~d}(\mu \mathrm{L})$} & \multicolumn{2}{|c|}{$2 \mathrm{e}(\mu \mathrm{L})$} & \multicolumn{2}{|c|}{$2 \mathrm{f}(\mu \mathrm{L})$} & \\
\hline & 50 & 100 & 50 & 100 & 50 & 100 & 50 & 100 & 50 & 100 & 50 & 100 & \\
\hline & \multicolumn{13}{|c|}{ Zone of inhibition in $\mathrm{mm}$} \\
\hline Proteus mirabilis & - & - & 10 & 22 & - & 15 & - & - & - & - & 13 & 14 & 31 \\
\hline Bacillus cerus & - & - & 13 & 18 & 15 & 17 & 13 & 15 & 10 & 16 & 11 & 17 & 29 \\
\hline Staphylococcus aureus & 12 & 17 & 17 & 22 & - & 14 & - & 12 & 15 & 21 & 14 & 18 & 28 \\
\hline Salmonella typhi & - & - & - & - & - & - & - & - & - & 16 & - & - & 38 \\
\hline Escherichia coli & 17 & 20 & 18 & 23 & 16 & 18 & - & 14 & - & - & 12 & 18 & 28 \\
\hline
\end{tabular}

\section{CONCLUSION}

In conclusion, we have presented a facile route to diol derivatives $\mathbf{2 a - h}$ starting from isocoumarin derivatives, $\mathbf{1}$. The synthesized diol derivatives showed good antibacterial activity against Staphylococcus aureus.

\section{REFERENCES}

1. S.O. Zcan, E.S. Ahin, M. Balci, Tetrahedron Lett. 48, 2151, (2007)

2. S.H. Lim, S.M. Hudson, Carbohyd. polym. 56, 227, (2004).

3. R.B. Tirodkar, R.N. Usganokar, Indian J.Chem. 10, 1060, (1972).

4. M. Odabas, Odabas_og lu, C, ig dem Albayrak a, Res it $\mathrm{O}^{\prime \prime}$ zkanca b, Fatma Zehra Aykan, Peter Lonecke, J. Mol. Struct. 840, $\overline{7} 1$, (2007).

5. F. Bihel, G. Quelever, H. Lelouard, A. Petit, C.A. da Costa, O. Pourquie, F. Checler, A. Thellend, P. Pierre, J.L. Kraus, Bioorgan. Med. Chem. 11, 3141, (2003).

6. S.Y. Kang, K.Y. Lee, S.H. Sung, M.J. Park, Y.C. Kim, J. Nat. Prod. 64 $683,(2001)$.

7. T.P. Thrash, T.D. Welton, V. Behar, Tetrahedron Lett. 41, 29, (2000).

8. E.C.Y. Woon, A. Dhami, M.F. Mahon, M.D. Threadgill, Tetrahedron 62 , 4829, (2006).

9. V. Subramanian, V.R. Batchu, D. Barange, M. Pal, J. Org. Chem. 70, $4778,(2005)$.
10. S. Syed Tajudeen, F. Nawaz Khan, Synth. Commun. 37, 3649, (2007).

11. Nitin T. Patil, F. Nawaz Khan, Y. Yamamoto, Tetrahedron Lett. 45, 8497, (2004).

12. F. Nawaz Khan, R. Jayakumar, C.N. Pillai, J.Mol.Cat. A-Chem. 195, 139, (2003).

13. F. Nawaz Khan, R. Jayakumar, C.N. Pillai, Tetrahedron Lett. 43, 6807, (2002).

14. V. R. Hathwar, P. Manivel, F. Nawaz Khan, T. N. Guru Row, Acta Cryst. E63, 03707, (2007).

15. V. R. Hathwar, P. Manivel, F. Nawaz Khan, T. N. Guru Row, Acta Cryst. E63, 03708, (2007).

16. V. R. Hathwar, Manivel, F. Nawaz Khan, T. N. Guru Row, CrystEngComm. 11, 284, (2009).

17. P. Manivel, V. R. Hathawar, K. Prabakaran, S. Mohana Roopan, F. Nawaz Khan, Acta Cryst. E65, o261, (2009).

18. P. Manivel, V. R. Hathawar, P. Nithya, R. Subashini, F. Nawaz Khan Acta, Cryst. E65, o254, (2009).

19. P. Manivel, S. Mohana Roopan, F. Nawaz Khan, J. Chil. Chem. Soc. 53, $1609,(2008)$.

20. S. Mohana Roopan, T. Maiyalagan, F. Nawaz Khan; Can. J. Chem. 86, 1019, (2008).

21. G. Grover, S.G. Kini, Eur. J. Med. Chem. 41, 256, (2006). 